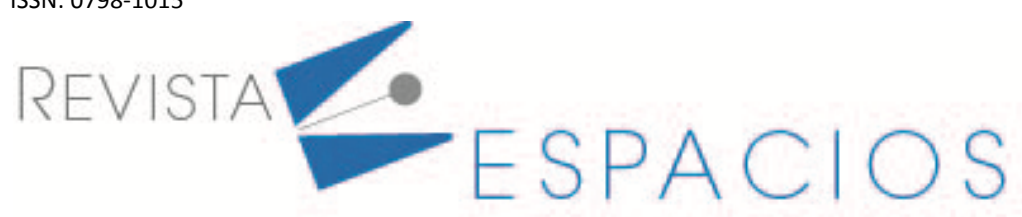

\title{
Geometría, tecnología y experiencia en la enseñanza de arquitectura
}

\section{Geometry, technology and experience in teaching architecture}

\author{
ESPINOZA, Felipe ${ }^{1}$ \\ RENDÓN, Jaluff I. ${ }^{2}$ \\ CHING, Johnson Y. ${ }^{3}$
}

\section{Resumen}

Se plantea la reforma del plan curricular y la forma de enseñanza de la arquitectura, sobre la base epistemológica de la producción de conocimientos, que se fundamenta en la práctica proyectual y donde la geometría tiene relevancia especial en la formación; se registra la exploración curricular con el propósito de desentrañar el desarrollo que tiene la geometría como articulador en la formación del arquitecto $y$, desde la práctica de la enseñanza, se realiza una experimentación de la geometría en un living lab, en el marco de la sostenibilidad.

Palabras clave: plan de estudios, geometría, proyecto espacial, ecosistema, bloques, prototipo.

\begin{abstract}
The reform of the curriculum and the way of teaching architecture is proposed based on the epistemological basis of knowledge production that is based on project practice, where geometry and experience have a special relevance in training; the curricular exploration is registered with the purpose of unraveling the degree of development that geometry has as an articulating entity in the training of the architect and, from the practice of teaching, an experimentation is carried out in a living lab mode, within the framework of sustainability.
\end{abstract}

key words: curriculum, geometry, space project, ecosystem, blocks, prototype.

\section{Introducción}

Cualquier construcción teórica sobre el que hacer de la arquitectura, debe partir de las nociones de epistemología de la arquitectura, la producción de conocimiento se fundamenta en el "proceso proyectual de la experimentación del espacio" para elaborar el proyecto arquitectónico como una forma más de conocimiento" (Muñoz, 2019).

El proceso proyectual es el resultado de la articulación de diferentes conocimientos con las técnicas e instrumentos con los que se desarrolla, siendo relevante la base geométrica que origina el espacio; sin embargo estas herramientas, sin excepción, actúan de forma efectiva y contundente cuando cumplen dos requisitos: el primero, articulando la forma de entender el desarrollo de proyecto y en segundo lugar, considerando el uso técnico para dicho desarrollo, de acuerdo con las tecnologías con las que se cuente (Correal, 2007).

\footnotetext{
${ }^{1}$ Docente. Facultad de Arquitectura. Universidad de Guayaquil. felipe.espinozao@ug.edu.ec. Orcid: 0000- 0003-3733-0823

${ }^{2}$ Docente. Facultad de Arquitectura. Universidad de Guayaquil. ivonne.rendonj@ug.edu.ec. Orcid: 0000-0003-4144-7979

${ }^{3}$ Docente. Facultad de Arquitectura. Universidad de Guayaquil. jhonson.chingc@ug.edu.ec. Orcid: 0000- 0002-2060-9628
} 
Para el efecto es necesario abordar algunas definiciones sobre: La epistemología de la arquitectura. La geometría. El desarrollo sostenible, la optimización de recursos y la tecnología. El funcionamiento de los ecosistemas y la teoría de sistemas. Y La enseñanza del proyecto arquitectónico.

\subsection{Epistemología de la arquitectura}

La epistemología de la arquitectura ha sido abordada desde diferentes campos y perspectivas; el campo psicológico con las fundamentaciones de los trabajos de Jean Piaget, el campo antropológico con los estudios de Levi-Strauss, Rapoport, Leroi-Gour, el campo diverso de la semiótica, con Noam Chomsky y desde luego, las combinaciones de los campos mencionados (Muntañola, 1974).

Sin embargo, hay un campo que poco se ha explorado para revitalizarlo, y que tiene que ver con la formación de las matemáticas y de la geometría; en relación con la física, este campo tiene mucho que ver con la forma como miramos el mundo, y es el campo donde se han generado más innovaciones.

Por mucho tiempo la enseñanza de la geometría en la formación de arquitectos e ingenieros, se fundamentó en la geometría clásica de Euclides; muy poco se ha explorado en las geometrías no euclídianas, mucho menos se ha explorado en las geometrías relacionadas con la física, donde se han encontrado nuevas experiencias, que han modificado los conceptos de geometría (Biagioli, 2017).

\subsection{Geometría}

Los griegos fundamentaron el concepto de geometría como medida de la tierra, explorando cinco formas perfectas que se llaman solidos platónicos, y los relacionaron con los elementos esenciales de la naturaleza, el tetraedro, el cubo, el octaedro, el dodecaedro y el icosaedro. Los griegos con Los Elementos de Euclides sentaron las leyes de la geometría con un afán muy básico de descubrir las leyes que rigen las formas de la naturaleza. El imperio euclidiano ha tenido poca modificación en siglos y la enseñanza de la geometría no ha sido la excepción.

Tapia (2013) menciona que ciertamente con un valor innovador la geometría de Einstein sobresale con la idea de que la gravedad es una propiedad geométrica del universo, finalmente se apoyaría en la geometría de Ricci

y de Riemann, su afirmación es demoledora cuando dice en el mismo artículo que la experiencia y los hechos reales desbordan a la geometría euclídea, dando paso a la geometría no Euclidiana.

Conforme a la ciencia moderna, ninguna experiencia corresponde sólo y exclusivamente a la geometría, sino a la geometría unida a la mecánica, óptica, etc. Pero por otra parte, la geometría debe preceder a la física, puesto que las leyes de la última no pueden ser expresadas sin el auxilio de la primera; aparece la geometría como precediendo lógicamente a toda experiencia y a toda ciencia experimental. Así se llegó a que, no sólo para los matemáticos y filósofos, sino también para los físicos de principios del siglo XIX, los fundamentos de la geometría euclidiana apareciesen como algo absolutamente inamovible (Tapia, 2013).

En el gran marco del avance de los estudios complejos de geometría no debemos dejar de mencionar las aportaciones del profesor Karl H. Pribram, de la Universidad de Stanford, con sus trabajos sobre el funcionamiento holonómico del cerebro (generación de imágenes espaciales), y los avances sobre el proceso holográfico de este órgano para configurar información espacial, que como vemos, tienen aplicaciones para desarrollar aprendizajes a través de sesiones holográficas interactivas (realidad virtual) en el computador utilizando lentes especiales que permiten manipular los objetos espaciales con punteros digitales (Pribram, 1981).

Muy pronto la educación de arquitectura utilizará estas herramientas de realidad virtual para analizar las bondades del proyecto arquitectónico en el proceso proyectual con mayor versatilidad que la actual enseñanza del taller de proyectos. No menos importante es examinar el papel y la función de la geometría con los nuevos 
medios tecnológicos. Por ejemplo, Souza y Luccas, (2015) mencionan que las posibilidades presentadas por el software NonEuclid le permiten ser utilizado como un recurso para enseñar Geometría Hiperbólica al abordar dinámicamente sus características, así como profundizar el conocimiento geométrico.

Por ello se torna vital el manejo de herramientas para el diseño arquitectónico en la formación del arquitecto. Desde la antigüedad el hombre ha buscado la manera de expresarse, mediante códigos como el lenguaje o el dibujo en arquitectura. En el dibujo ha predominado la utilización de la geometría en arquitectura, aplicada directamente a la concepción y construcción de los objetos arquitectónicos; desde la elaboración de planos hasta la elaboración de los modelos tridimensionales o maquetas.

El arquitecto italiano Filippo Brunelleschi unió las matemáticas y la geometría con las proyecciones mentales artísticas de la arquitectura para crear la perspectiva lineal. Esta técnica todavía se ensena en las facultades de arquitectura, por la precisión de su escala medible, sin embargo, nuevas técnicas de representación del espacio están dominando la profesión, sin que nos demos cuenta.

Debe añadirse, que en los colegios de educación media en Ecuador todavía se enseña Geometría de una manera tradicional, que adolece de la falta de exploración en los enfoques de geometría fractal, geometría afín, geometría topológica de Leibniz, Euler, Hobbes, Riemann, Mandelbrot, así como su ajuste a las nuevas tecnologías, etc. Por ello, tanto profesores y estudiantes tienden a reproducir los modelos de geometría que de algún modo conocieron y experimentaron.

Por ello, la geometría, entendida como un medio para desarrollar la capacidad de imaginar el espacio en la mente, y permitir la expresión dibujada de los volúmenes con los que aquél se define y limita, es la parte clave en la que debemos enfocarnos.

\subsection{El desarrollo sostenible, la optimización de recursos y la tecnología}

Las actividades del hombre han causado importantes impactos sobre la naturaleza, y este ha tratado de encontrar formas de minimizar estos impactos, a través modelos capaz de describir correctamente la realidad, en búsqueda de la optimización de los recursos planetarios. Puede haber errores: los primeros modelos establecidos por el Club de Roma (asociación de investigadores preocupados por los límites de crecimiento de la humanidad), aspiraban a describir la evolución del ecosistema constituido por la tierra en sí misma. Pero se ha comprobado que los estados previstos se apartan mucho de lo que ha ocurrido en la realidad. Sin embargo, el Club de Roma apuesta que el desarrollo sostenible del crecimiento de la humanidad podrá sustentarse apoyando el desarrollo en los avances tecnológicos. Por su parte O'Neill de la Universidad de Leeds propone un sistema de 11 objetivos sociales, relacionados con 7 indicadores de bio capacidad, para encontrar un modelo de crecimiento sostenible (Torres, 2019). De alguna manera desde el 2015 los Objetivos de desarrollo sostenible son materia de discusión en los estados y en la academia, y han dado un lugar prominente a la tecnología.

La exploración del desarrollo sostenible en la educación conlleva indudablemente a examinar el modelo IPAT, propuesto en la década de los 70 por Paul Ehrlich, John Holdren, and Barry Commoner que ilustra cómo la población (P), la riqueza (A) y la tecnología (T) han actuado como las fuerzas clave en el desarrollo sostenible, es un recordatorio útil de que la población, el consumo y la tecnología ayudan a dar forma a nuestro desarrollo, sobre todo para la optimización de recursos (Ehrlich \& Holdren, 1971).

Al respecto el profesor Maddison ha ilustrado como el empleo de la tecnología ha conducido al desarrollo económico de civilizaciones, jugando un papel importante en el desarrollo, y en la educación (Ferran, 2016). Por lo que la formación del arquitecto requiere incorporar el uso integrado del ambiente; desde esa perspectiva se pretende aportar una ayuda desde la docencia de la arquitectura para la "construcción de un meta modelo" de plan de estudio donde el graduado sea respetuoso con el ambiente y confortable para el ser humano. El 
metamodelo permitiría formular macro-áreas de conocimiento, incorporando la tecnología para lograr la optimización de recursos, el manejo y ahorro de energía, disminución de desechos y costos.

\subsection{El funcionamiento de los ecosistemas y la teoría de sistemas}

La educación en arquitectura ha tenido importantes avances en las universidades del primer mundo y los living lab han encontrado suficiente fundamentación en la ecología y esta, a su vez, nos familiariza con la noción de ecosistema: conjunto complejo constituido por seres vivos, el medio natural en el que viven y sus relaciones mutuas. Un bosque, por ejemplo, es un ecosistema que no puede explicarse por sí sólo a partir del estudio minucioso de las especies que lo componen. Únicamente una visión global, que considere las interacciones del bosque con su entorno (condiciones climáticas, naturaleza del suelo, efectos de polución, criterios de tala y repoblación, etc.), permitirá establecer un modelo para estudiar la evolución general del bosque.

Pero los ecosistemas llegaron lejos con Karl Ludwig von Bertalanffy quién propuso en 1932, la teoría general de sistemas. La teoría general de sistemas se propone sobrepasar el estudio individual de los componentes y procesos, añadiendo una visión global de la organización que permite el funcionamiento del conjunto. Además de los métodos matemáticos empleados para analizar los sistemas, nuevas disciplinas han contribuido enormemente al desarrollo de los sistemas, entre las más importantes se puede citar la cibernética, la teoría de la información, teoría de los juegos, etc. (Martín, 2011).

La teoría de los sistemas aparece actualmente como una disciplina que agrupa las diferentes ramas de la ciencia, contrarrestando la creciente especialización en las propuestas de proyectos individuales, que tiende a alzar los muros cada vez más altos entre individuos y entredisciplinas, la idea es que las fortalezas de los individuos se mezclen para procurar una producción colectiva. Como todo experimento, es necesario ponerlo a prueba en un sitio especifico, para tal efecto se utilizaron los criterios de los entornos de aprendizaje denominado Living Lab, iniciados formalmente por el profesor William J. Mitchell del Massachusetts Institute of Technology (MIT), quien sostiene que los living Labs son entornos de investigación que sirven para involucrar a los usuarios en la innovación y el desarrollo, para detectar, crear prototipos, validar y refinar soluciones complejas en contextos de la vida real múltiples y en evolución, bajo el concepto de eficiencia de los ecosistemas, estos entornos sirven también para monitorear la eficacia de los modelos de aprendizaje (Eriksson et al., 2006).

\subsection{La enseñanza del proyecto arquitectónico}

La educación superior es fundamentalmente clave para un futuro sostenible; cuenta con las herramientas necesarias para desarrollar nuevas ideas y una sociedad activa que participa en la experimentación para una vida sostenible (Hugo et al., 2018). Tradicionalmente, los cursos de educación superior en el área de ciencias exactas, incluida la ingeniería, presentan la enseñanzade la geometría en su plan de estudios como una materia básica para la formación de los estudiantes (Silva et al., 2018).

La enseñanza del proceso proyectual solo se fundamenta como un campo de experimentación de prácticas temáticas para resolver problemas de espacio y forma arquitectónica, que permita mediante prueba y error producir planos técnicos que se utilizaran en la construcción de la obra. La enseñanza del proceso proyectual ha caído en un mecanicismo sin consideraciones conceptuales acerca de la experiencia geómetra como punto de partida, como un modo de producir y construir conocimientos. Este mecanicismo se puede observar cuando no se consideran los algoritmos que permiten construir formas espaciales, y que dicho sea de paso seadoptan ciegamente los contenidos de los programas digitales para diseñar, sin considerar el fortalecimiento del pensamiento geómetra digital que debe ser propiciado por los programas curriculares de arquitectura (Pagel, 2008). 
Hoy los estudiantes y profesionales de la arquitectura utilizan diversos lenguajes de software para la representación del espacio, no obstante, dicha tecnología requiere mucho tiempo para dibujar, y mover piezas, liberando poco tiempo para las tareas específicas de la creación arquitectónica, por lo que no hemos alcanzado la máxima expresión de la tecnología en estos lenguajes al servicio de la creación en sí misma. La planificación educativa es una de las etapas desafiantes, pero debemos compararla con las grandes empresas que hacen su planificación con el objetivo de llegar a millones de personas y el maestro es responsable de observar a sus estudiantes y encontrar las necesidades de sus clases para que les haga pensar (Guatura \& Romão, 2015).

La generación de una teoría sistémica de la geometría simplificaría enormemente la construcción espacial, ya que permitiría la formulación de algoritmos, que a partir de unas mínimas reglas genera un universo de posibilidades, por ejemplo, a partir de un algoritmo simple o en combinación produciría estructuras espaciales complejas. Por lo tanto, el papel del pensamiento geómetra es descubrir y relacionar esos algoritmos, para ser utilizados por computadoras, personas, herramientas mecánicas, etc. La tarea es introducir el pensamiento algorítmico de geometría digital, y muy pronto utilizar la realidad virtual en el proceso proyectual. Desde esta perspectiva se pueden aportar respuestas empíricas al problema del conocimiento y su desarrollo.

Debe indicarse, que la enseñanza de proyectos, no ha sido modificada hace mucho tiempo, manteniéndose el modelo tradicional de un solo profesor para el taller, cuando cada vez más se ve la necesidad de articular diferentes conocimientos y especialidades, siendo necesario incorporar varios profesores de distintas especializaciones en el laboratorio de proyectos.

Finalmente se puede concluir que el objetivo de este trabajo tiene que ver con la necesidad de incorporar a la geometría como disciplina articuladora del curriculum de arquitectura y la necesidad de explorar un nuevo pénsum de estudios, sabiendo que el fortalecimiento del pensamiento geómetra-digital contribuye enormemente a enriquecer el proceso proyectual como una forma de generación de conocimiento. Visto así, el pensamiento geómetra-digital en el proceso proyectual, abrirá nuevos caminos para la solución y transformación de los problemas de la sociedad. La geometría entendida como el medio para desarrollar la capacidad de imaginar el espacio en la mente del estudiante, para que luego sea capaz de representar el espacio dibujado con muchas técnicas, según lo señala la Unión Internacional de Arquitectos UIA y la UNESCO a través de la Carta de la Formación de la Arquitectura, retomadas por el Proyecto Tuning de Latino América: "Habilidad de percibir, concebir y manejar el espacio en sus tres dimensiones y en las diferentes escalas".

\section{Metodología}

Para abordar la necesidad de reforma del plan curricular y la forma de enseñanza de la arquitectura se plantearon dos aspectos básicos para el análisis; la exploración curricular con el propósito de desentrañar el desarrollo que tiene la geometría como articulador en la formación del arquitecto, y la demostración del nivel de manejo de la geometría con los recursos tecnológicos que se disponen a través de un experimento de living lab aplicado a un proyecto de arquitectura que participó en el concurso del Banco Mundial denominado Resilient Homes Design Challenge (2018). Para el efecto se examinará el plan de estudios de la Facultad de Arquitectura de los años 2013, 2014, muy similares, comenzando con la revisión de los sílabos, los contenidos de las asignaturas que contienen los aspectos relacionados con la geometría.

Para lograr tal cometido se analizan las asignaturas del plan de estudios, correspondientes a las áreas de diseño, técnica, expresión, social y optativas, relacionadas con la formación geómetra, así como los contenidos, créditos, y horas: 
Para el primer nivel: Diseño Básico (Elementos De La Composicion), Edificaciones 1 (Materiales De Construcción), Dibujo Arquitectónico, Análisis Arquitectónico, Matemáticas, Medios De Expresión, Geometría Descriptiva, Cad 1.

Para el nivel 2: Proyectos 1 (Composición Arquitectónica), Edificaciones 2, (Materiales De Construcción), Dibujo Arquitectónico 2, Ingles, Composición 1, Matemáticas 2, Medios De Expresión 2, Física, Perspectiva , Cad 2.

Para el tercer nivel: Proyectos 2 (Composición Arquitectónica) Edificaciones 3 (Procesos Constructivos) Medios De Expresión Artistica 3Teoria E Historia De La Arquitectura, Estructuras 1 (Estática), Metodologia De La Investigacion1, Ingles 2.

Para el cuarto nivel : Proyectos 3 (Diseno Arquitectonico), Edificaciones 4 (Mejoramiento De Terrenos), Tecnica De Presentacion Arquitectonica, Estructuras 2 (Resistencia De Materiales),Topografia.

Para el quinto nivel: Proyectos 4 (Diseño Arquitectónico), Diseno Urbano 1, Edificaciones 5 (Hormigon Armado), Estructuras 3 (Análisis Estructural), Instalaciones 1, Teoria E Historia De La Arquitectura 3, Teoria Urbanistica 1, Arquitectura Bioclimática, Adm. De Obras Y Proyectos, Ingles 4.

Para el sexto nivel: Proyecto 5 ( Anteproyecto Arquitectónico 1), Diseño Urbano2, Edificaciones 6 (Prefabricado De Hormigon), Estructuras 4 (Diseno Estructural), Instalaciones 2, Analisis Arquitectonico 2, Teoria Urbanistica 2, Paisajismo, Arquitectura Bioclimatica, Mecanica De Suelos, Topografia 2, Desarrollo Sustentable.

Para el séptimo nivel: Proyectos 6 (Anteproyecto Arquitectonico 2), Edificaciones 7 (Prefabricados Metalicos), Analisis De Costo Y Presupuesto, Diseno Urbano 3, Ambiente Y Ciudad, Estetica, Acustica Y Luminotecnia, Fiscalizacion De Obra, Construccion De Estructuras De Cana Y Bambu, Legislacion Ambiental, Desarrollo Urbano Sustentable 1.

Para el octavo nivel: Proyectos 7 (Proyecto Arquitectonico), Edificcaiones 8 (Patologia En La Construccion), Instalaciones 3, Diseño Urbano 4, Infraestructura Urbana, Normativa Urbana, Peritaje De Obras, Desarrollo Urbano Sustentable 2.

Para el nivel 9: Proyectos 8.

Para el nivel 10: Trabajo De Proyectos De Grado.

Estos análisis permitirán agrupar los resultados cuantitativos con el objeto de decantar el peso de la formación geómetra en el plan de estudio de arquitectura y naturalmente, su impacto en el proceso proyectual. (Ver Grafico 1)

Para explorar el manejo de la geometría y las aplicaciones tecnológicas por parte de los docentes y estudiantes de la Facultad de Arquitectura y Urbanismo de la Universidad de Guayaquil, se llevó a cabo un experimento bajo la modalidad de living lab como parte sustancial del proyecto de investigación denominado "Vivienda Económica con tecnología de paneles". El experimento consistió en la elaboración de un prototipo digital de vivienda económica y se llevó a cabo en el aula 402 con autorización del Consejo de Facultad oficio 911-DEC-ARQ-2016, con la participación de 6 profesores y 32 estudiantes de los cursos de 1 al 10 semestre. Para organizar el proceso de fabricación del prototipo se consideró la línea de producción Ford, dividiendo las áreas de producción en 16 bloques de desarrollo, que luego deberían conectarse con sus resultados, el proceso debía monitorearse a través de un sistema de indicadores no mayor de 10, para poder observar los puntos fuertes y débiles del desarrollo de la producción del prototipo, poniendo especial énfasis en la experiencia de geometrización del espacio a través del manejo de los software utilizados para diseño. 
Como todo prototipo de fabricación se dividió el proyecto en 16 partes, a las que llamamos bloques de desarrollo, constituidos por:

1.-Normas de colores, 2.-Kit embalaje, 3.-Manual de construcción, 4.-Instalaciones, 5.-Resinas y pegantes, 6.Economía de materiales, 7.-Memoria técnica, 8.-energía y atmosfera, 9.-ubicación y análisis de sitio, 10.materiales y recursos, 11.-Eficiencia energética, 12.-Calidad de ambiente interior, 13.-Calidad y servicio, 14.Parámetros de confort, 15.-Aspecto socio-económico-cultural, 16.-Fabricación del prototipo.

Los indicadores que se plantearon fueron; 1.-Desarrollo de algoritmos, 2.-Resolución de problemas y grado de innovación, 3.-Planificación y previsión de tareas entre docentes y estudiantes, 4.-Grado de coordinación e interdependencia de los componentes, 5.-Utilización de herramientas tecnológicas, 6.-Grado de ajuste, 7.Focalizador del atractor, 8.-Grado de velocidad en la investigación de campo, 9.-Defensa de las ideas y grado de desempeño, 10.-Desarrollo de una plataforma colaborativa o registro.

El experimento tuvo la finalidad de proponer la utilización de un entorno de aprendizaje denominado living labs que son entornos de investigación para involucrar a los usuarios en la innovación y el desarrollo, para detectar, crear prototipos, validar y refinar soluciones complejas en contextos de la vida real múltiples y en evolución, bajo el concepto de eficiencia de los ecosistemas, con el objeto de monitorear el modelo de aprendizaje y por otro lado, producir un prototipo digital de vivienda requerido con los más altos estándares exigidos para la participación del concurso del Banco Mundial Resilient Homes Design Challenge (2018). Pero además, permitió analizar como la geometría y el uso del espacio es el eje que enlaza todo el proceso de donde se derivan las propuestas de mejora del plan de estudios de arquitectura en la práctica de proyectos.

La participación en el concurso del Banco Mundial, se presentó como una situación ideal para desarrollar el experimento, ya que parte de los requisitos exigía la metodología de trabajo colaborativo para alcanzar varios criterios de evaluación de la propuesta arquitectónica, a saber: Resistencia (25\%), Diseño (20\%), Rentabilidad (15\%), Construcción (15\%),Sostenibilidad (15), y Presentación (10\%). Aspectos estos que se relacionaban con el experimento: lograr una propuesta sostenible de bajo costo, desarrollada en un ambiente colaborativo, permitiendo dejar ver la necesidad de adecuar y modelar nuevas formas de enseñanza de la geometría en el proceso proyectual, utilizando modalidades de enseñanza living lab.

Para complementar esta tarea se debía realizar un seguimiento muy específico sobre las fortalezas de los estudiantes, sus diferentes destrezas y capacidades, y sobre el grado de dominio de los softwares que utilizaran para el desarrollo del proyecto. La supervisión del avance del proyecto en el laboratorio debe estar a cargo de un número de profesores que controlen el desarrollo de los bloques, quienes monitorearan los procesos y resultados, siendo necesario estructurar un sistema de indicadores (no mayor a 10) que permita monitorear el avance del proyecto y los bloques de desarrollo del proyecto (no mayor a 16). Una vez alcanzado cierto grado de desarrollo se comienza a "intersectar los bloques", encontrándose los puntos fuertes y débiles del proyecto. En ese proceso aparecen innovaciones insospechadas que ameritan su registro para los ajustes del proyecto y redacción de los artículos.

En la fase final de producción del prototipo, se empezó a unificar toda la producción de los resultados de los bloques en una plataforma digital, que la gerencia un profesional o profesor conjuntamente con un estudiante, quienes integrarán los productos, en discusión entre alumnos y profesores; llegado a este punto es necesario implementar una estrategia avanzada que incluye los foros de profesionales que dominan las disciplinas relacionadas con los bloques de conocimiento mencionados. Con esta dinámica se encuentran ajustes importantes que permiten avanzar profesionalmente en el proyecto. Estos ajustes son tratados con los estudiantes y luego llevados a la gerencia de la plataforma digital quien va integrando todo el proceso y discutiendo con los estudiantes los avances logrados. 
El objetivo de este trabajo es el análisis de dos procesos integrados e inclusivos que se requieren en la formación del arquitecto, el examen del plan de estudios que permita encontrar hallazgos para estructurar mejoras o requerimientos nuevos en la enseñanza de la geometría en las asignaturas, en la dosificación suficiente o insuficiente que se asigna para la formación espacial, examinando el papel de las nuevas tecnologías que se utilizan en la formación geómetra del arquitecto para desarrollar el potencial espacial del estudiante, con más competencia y profesionalismo.

Al mismo tiempo la implementación del living lab, pone a prueba una nueva forma de enseñanza de la arquitectura, una nueva experiencia, una nueva práctica de enseñanza en la elaboración de proyectos, que permitiría superar las carencias en la formación de arquitectos en la elaboración de proyectos, el experimento tiene el propósito de encontrar hallazgos relativos a la forma que los estudiantes trabajan en un laboratorio integrando conocimientos en tiempo real, observando la eficacia de la construcción de conocimientos colectivos, la intersección de áreas o bloques de conocimiento al mismo tiempo y en un mismo lugar, que permita actuar rápido en el diseño, observando las limitaciones o bondades que tienen al utilizar y transformar las tecnologías de formación geómetra para resolver los problemas de diseño.

Visualizar, dibujar y representar el espacio, es una parte inseparable del proceso de diseño, los talleres de proyectos y el resto del plan de estudios requieren preparar un instrumento que le da a los alumnos la posibilidad de presentar y comunicar sus propuestas frente a sus pares, sus docentes y frente a sí mismos.

La representación arquitectónica hacia el mundo digital es un proceso progresivo que viene avanzando año tras año, pero si no estamos preparados conceptualmente, preparando el pensamiento digital geómetra, no la podríamos aprovechar.

\section{Resultados}

La malla curricular de la Facultad de Arquitectura de la Universidad de Guayaquil 2013-2014, (ver FIGURA 1), no presenta evidencia de elementos articuladores sostenidos a lo largo de la carrera, que contribuyan a abordar de forma eficaz la producción de conocimientos en el proceso proyectual como una forma de conocimiento. La formación de Geometría en la Facultad de Arquitectura de la Universidad de Guayaquil no tiene la relevancia como un elemento articulador que permita consolidar una formación geómetra en las asignaturas que configuran el plan de estudios.

Por otro lado, en la Facultad de Arquitectura de la Universidad de Guayaquil se registra muy poca investigación para fundamentar su enseñanza como un elemento innovador cuando se combina con otras ciencias que produzcan nuevas experiencias en el proceso proyectual de la experimentación del espacio, pudiéndose observar una notable carencia en la evaluación del indicador 5 (Utilización de herramientas tecnológicas), para la fabricación de la propuesta que se presentó en el concurso del Banco Mundial. Debe indicarse que la geometría entendida en el marco de los axiomas euclidianos posiblemente registre pocas innovaciones en arquitectura, de hecho no se registran muchas patentes relacionadas con "sistemas espaciales constructivos" según registros de la Dirección de Investigación de la Universidad de Guayaquil. 
Figura 1

Malla de arquitectura 2013-2014

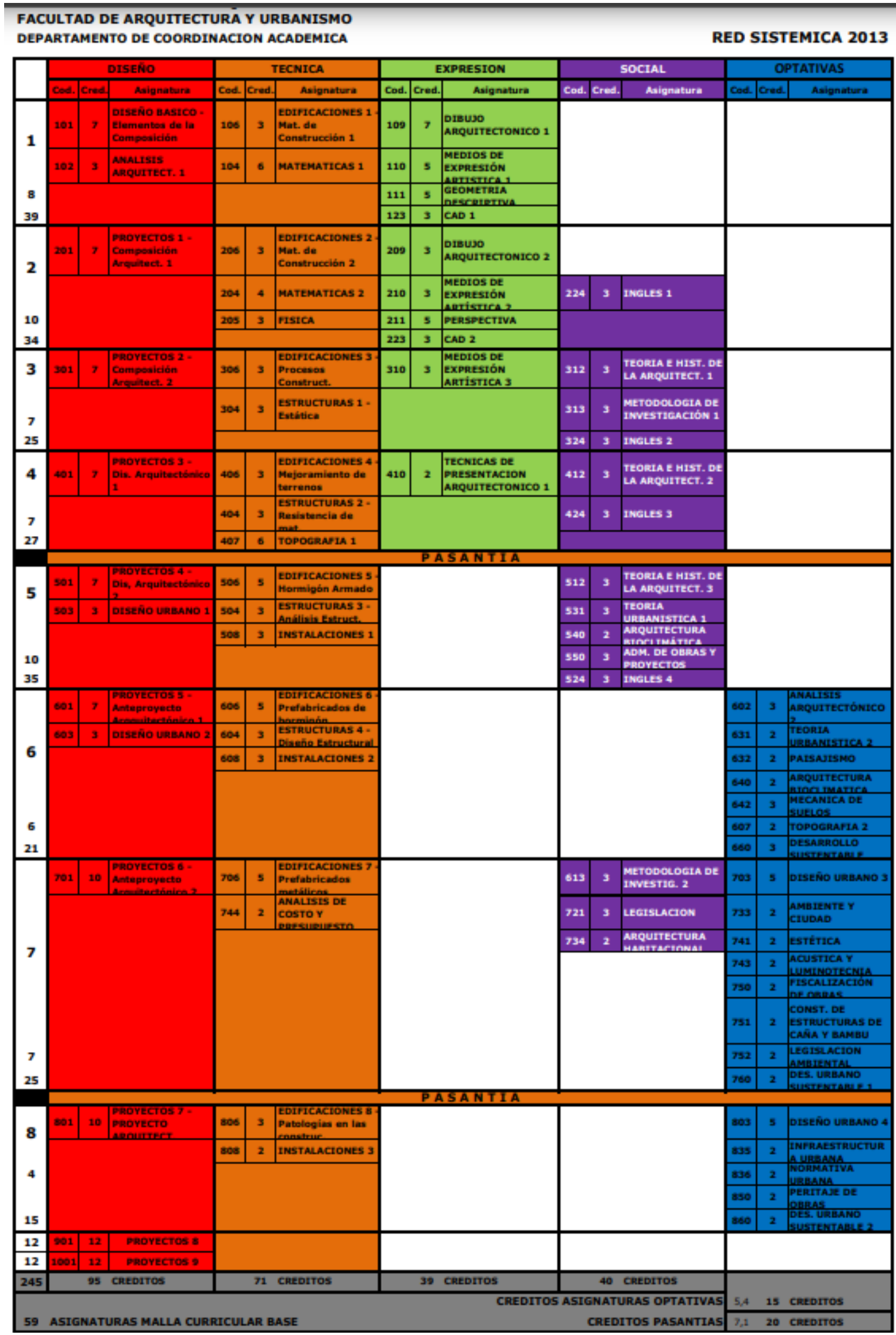


Los resultados del examen de la malla curricular, ofrecen evidencia de la escasa formación geómetra del estudiante, tan solo el $14 \%$ del pensum constituye la formación de geometría, focalizadas en el área de expresión. (Ver Figura 2). El resto del plan de estudios lo constituyen el taller de proyectos que se circunscribe en el 32\%: el área técnica, el $28 \%$, el área social el $13 \%$, y las optativas, el $13 \%$.

Hemos asignado al estudiante sólo dos años de formación en geometría cuando esta disciplina es el ADN de la profesión, pero sobre todo resalta una educación tradicional en geometría; no se observa un desarrollo sostenido de la formación geómetra en todo el plan de estudios, como tampoco la conexión de geometría y las nuevas tecnologías con los modelos digitales 3D, con la proyectación arquitectónica y el sistema constructivo. Tal como se presenta la "formación geómetra" en arquitectura no hemos llevado esta disciplina a sus límites; geometría de la transformación, geometría topológica, mecánica geométrica, aglomeraciones geométricas, geometría fractal de Mandelbrot (The Fractal Geometry of Nature). Posiblemente esta conexión permita, en un futuro, sustentar el proyecto de una forma más sólida.

Es muy importante utilizar estándares nacionales y/o internacionales para poder comparar el comportamiento de los patrones de contenidos de geometría y física de la malla curricular de la Facultad de Arquitectura de la Universidad de Guayaquil (UG) con la malla curricular de la Escuela de Arquitectura del Instituto Tecnológico de Massachusetts, como ejemplo. EI MIT, dentro de su plan de estudios (ver Figura 3), desarrolla la geometría con una perspectiva distinta en el área de computación, investiga métodos de diseño arquitectónico y desafía los límites de la tecnología actual, así como la enseñanza y la práctica del diseño convencional. Se centra en el desarrollo de herramientas innovadoras de cómputo, los procesos de diseño y teorías, y la aplicación de éstos en respuestas creativas y socialmente significativas a los problemas de diseño difíciles. La Facultad trabaja en:

-Visualización, video y animación

-Tecnologías de prototipado rápido y CAD / CAM

-Forma de representación y dar forma a la síntesis

-La construcción de modelado de información (BIM )

-Diseño generativo y paramétrico

-Estudios críticos de las tecnologías digitales y de la información

-Desarrollo de software / hardware de herramientas avanzadas para el diseño espacial

-Aplicación de la tecnología de diseño a los problemas reales de diseño.

A pesar de la evolución tecnológica en la representación del espacio, las grandes universidades del primer mundo siguen respetando y manteniendo la esencia del modelo empleado tradicionalmente en las Escuelas de Arquitectura desde la época de Vitruvio, hace 2.000 años, en su tratado de arquitectura, pasando por la Bauhaus, Mies van der Rohe, Ludovico Quaroni, etc., en cuanto a la importancia estratégica de la formación geómetra del arquitecto. Lo único que ha cambiado es la tecnología, pero la importancia de la formación geómetra no ha cambiado y vemos que las tecnologías digitales 3D se presentan a todo lo largo del plan de estudios del MIT, para formar un área de pensamiento digital.

Como resultado de esta comparación se evidencia un déficit en la formación geómetra de la malla de la Facultad de Arquitectura de UG, siendo crítico la ausencia del área de formación del pensamiento digital, que representa la parte más innovadora en la formación geómetra que debe tener la formación del arquitecto (Candela, 1975). 
Figura 2

Peso de las áreas académicas

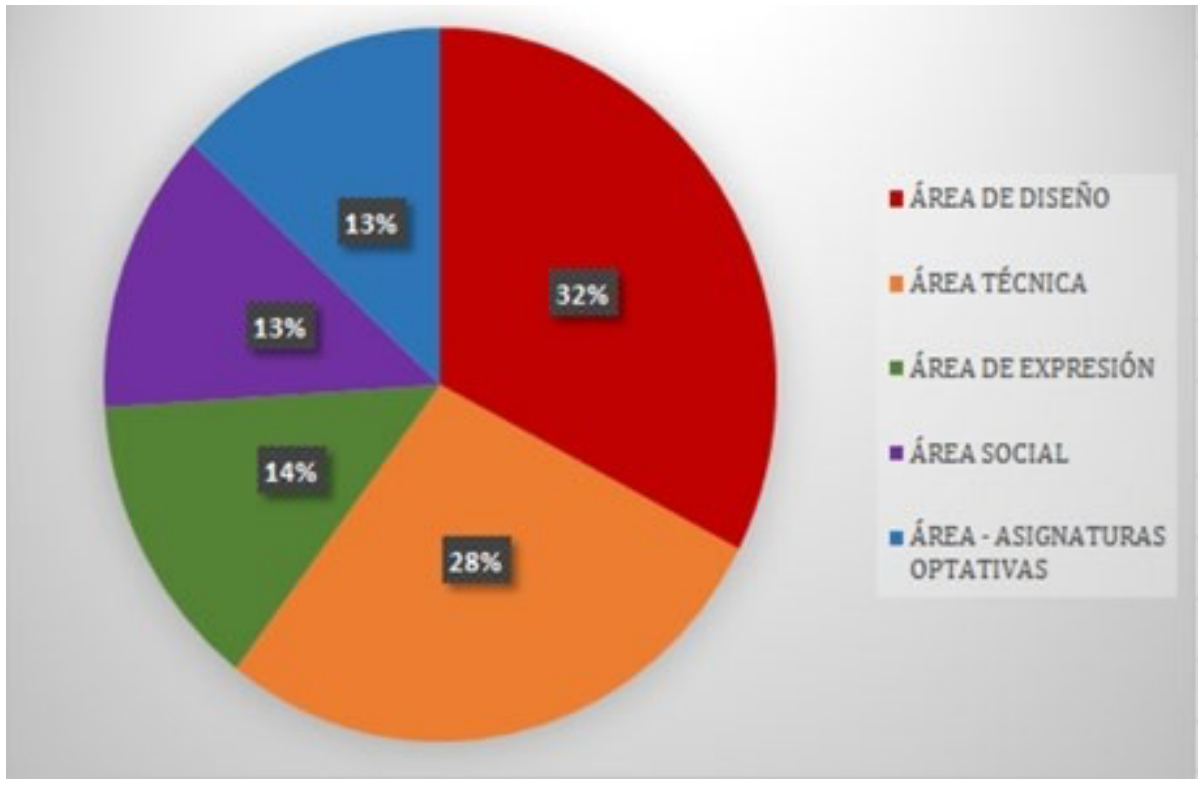

Fuente: Programa curricular de la Facultad de Arquitectura 2013-2014

Figura 3

Estructura General del pénsum del MIT

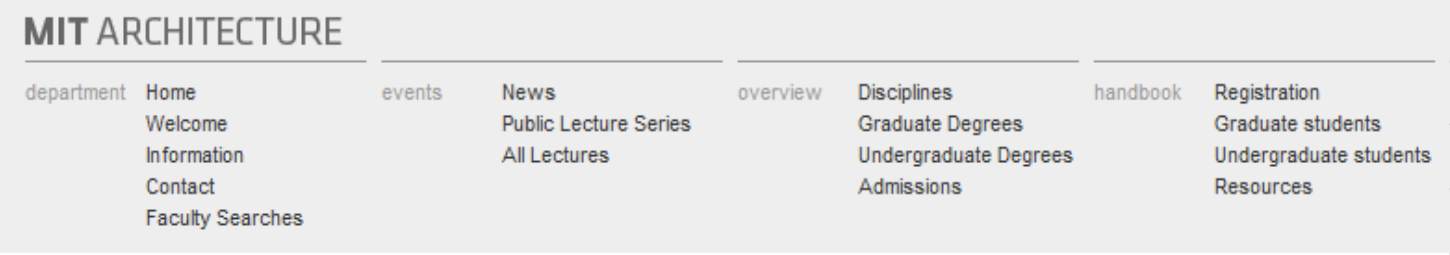

Faculty Searches

\begin{tabular}{|c|c|c|c|}
\hline \multicolumn{2}{|c|}{ Architectural Design } & \multicolumn{2}{|c|}{ Art Culture + Technology } \\
\hline events & News & events & News \\
\hline & Lectures & & Lectures \\
\hline \multirow{9}{*}{$\begin{array}{l}\text { program } \\
\text { degrees }\end{array}$} & Overview & \multirow[t]{3}{*}{ program } & Overview \\
\hline & & & Faculty Research \\
\hline & $\begin{array}{l}\text { SMArchS } \\
\text { MArch }\end{array}$ & & Resources \\
\hline & BSA & \multirow[t]{2}{*}{ degrees } & SMACT \\
\hline & & & BSA \\
\hline & Subjects & \multirow{2}{*}{\multicolumn{2}{|c|}{ Subjects }} \\
\hline & \multirow{2}{*}{ Projects } & & \\
\hline & & \multicolumn{2}{|r|}{ Projects } \\
\hline & Publications & & \\
\hline & People & \multicolumn{2}{|r|}{ People } \\
\hline
\end{tabular}

People

\begin{tabular}{ll} 
Building Technology \\
events & $\begin{array}{l}\text { News } \\
\text { Lectures }\end{array}$ \\
\hline program & $\begin{array}{l}\text { Overview } \\
\text { Research Topics }\end{array}$ \\
degrees & $\begin{array}{l}\text { PhD } \\
\text { SMBT } \\
\text { SMArchS } \\
\text { BSA }\end{array}$ \\
\hline & Subjects \\
\hline & Projects \\
\hline & Publications \\
\hline & People
\end{tabular}

\begin{tabular}{l}
$\quad \operatorname{login} \rho$ \\
\hline Subjects \\
\hline Projects \\
\hline People \\
\hline
\end{tabular}

Research

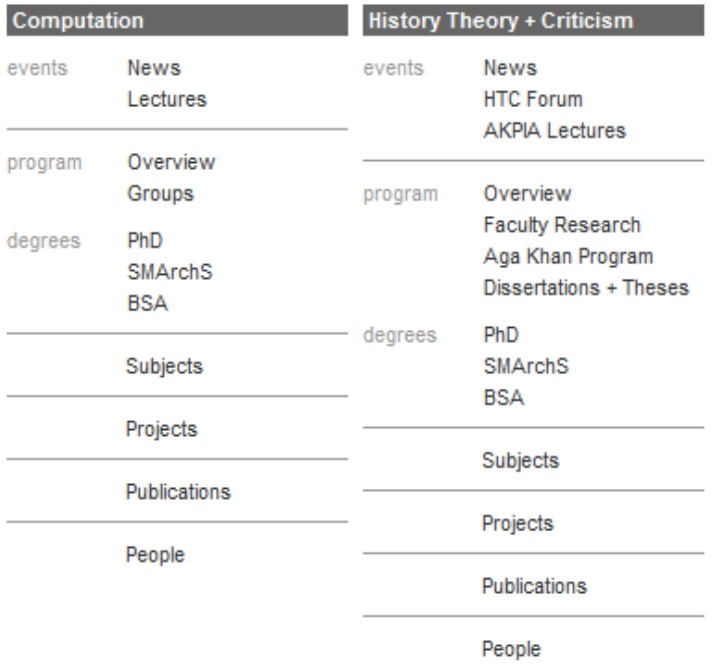

Respecto a los resultados de la matriz de indicadores de monitoreo y componentes de desarrollo en el experimento living lab que se desarrolló para participar en el concurso del Banco Mundial, se puede plantear 
que los estudiantes fueron agrupados de acuerdo a sus fortalezas en cada uno de los 16 bloques, luego de una fase inicial de desarrollo, encontrándose diferencias de resultados, de hecho hay que decir que no todos los bloques tienen una eficacia o potencia máxima de manera uniforme, parece ser que el sistema tiene un patrón de comportamiento inicial, intermedio y final, y las energías y relaciones no se distribuyen de forma pareja. Resulta paradójico ya que se ofreció a los estudiantes la libertad para organizarse en los bloques de acuerdo con sus fortalezas.

En el desarrollo de los bloques, aparecieron los estudiantes especialistas, en cada población o bloque presentaron auto reproducciones y autoregulaciones como los ecosistemas y se pudo notar la variación de conocimientos de los diferentes estudiantes, unos más diestros que otros en obtener soluciones ante los problemas presentados, notándose una mayor diversidad de destrezas que cuando asumen los trabajos individuales. Es importante enfatizar estos resultados porque son los equipos interdisciplinarios los que sobreviven mejor en condiciones de extrema producción(Sanders, 2005), si no miremoslos equipos que serán enviados a la misión Mars One (biólogo, medico, botánico, ingeniero mecánico) (Kauffman, 2015).

Respecto a la medición de los indicadores, se encontraron serias deficiencias en la utilización de herramientas tecnológicas (4.8/10) y en desarrollo de la plataforma colaborativa (4.0/10), mientras que los indicadores relacionados con los aspectos creativos, resolución de problemas, planificación, focalización de atractores, velocidad de la investigación y argumentos estuvieron mejor posicionados. Según estos resultados se encuentran hallazgos prometedores que justifican la necesidad de implementar cambios en el plan de estudios de arquitectura el área de pensamiento digital e implementar la modalidad living lab en la enseñanza del taller de proyectos y de producción de prototipos que optimice los recursos humanos y materiales.

Mientras que los bloques más destacados se orientan al aspecto constructivo, como el manual de construcción, instalaciones, economía de materiales, energía y atmosfera, calidad del ambiente interior, calidad de servicio y fabricación del prototipo. El manual de construcción y la fabricación de prototipo presentó soluciones austeras en el concurso del Banco Mundial, evidenciando poca versatilidad en el manejo de la geometría, ello conduce necesariamente ha actualizar el pénsum de geometría y de otras asignaturas afines que introduzcan nuevas geometrías vinculadas con las tecnologías digitales 3D, que potencien la proyectación arquitectónica y el sistema constructivo.

En evaluación de los indicadores, la utilización de herramientas tecnológicas y plataforma colaborativa alcanzo bajo rendimiento, indicadores que jugaron un papel importante a la hora del concurso. Ver figura 4 "Resultad simplificados".

Por el lado de los bloques de desarrollo, encontramos competencias bajas en la construcción de la memoria técnica (5.4/10), y los aspectos relacionados con los detalles constructivos, como colores, kit de embalaje, resina y pegamento (5.8/10), materiales y recursos, eficiencia del agua, y otros temas relacionados con los bloques contextuales como aspectos socio culturales (5.9/10), parámetros de confort (5.7/10), y análisis de sitio.

Las observaciones realizadas en el proceso living lab, con el trabajo de los bloques, fortalecen el modelo de enseñanza colaborativo, siendo clave la combinación y énfasis de desarrollo de los bloques, si bien es cierto avanzamos en unos, pero no en otros. Es complejo alcanzar buenos resultados en todos los bloques, no obstante, debemos progresar descubriendo teorías parciales para desarrollar combinaciones posibles de los bloques, para tener resultados más eficaces que permitan evolucionar la enseñanza de la arquitectura a través de estos nuevos entornos de aprendizajes. 
Figura 4

Resultados simplificados

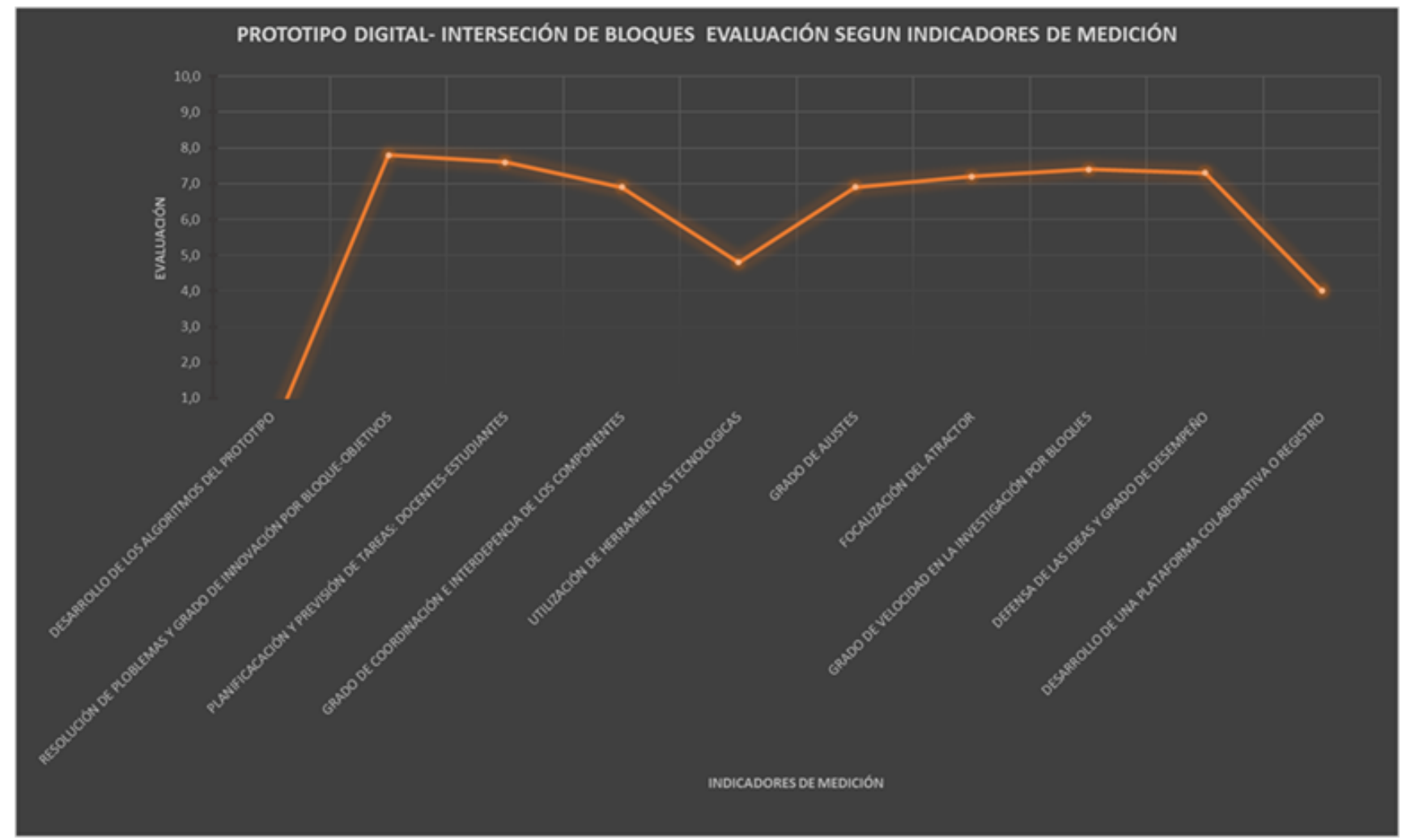

En el componente tecnológico de manejo de software de manera desagregada se encontraron niveles básicos de dominios (VER FIGURA 5). Dominio de software, estudiantes de arquitectura.

Figura 5

Dominio de software, estudiantes de arquitectura

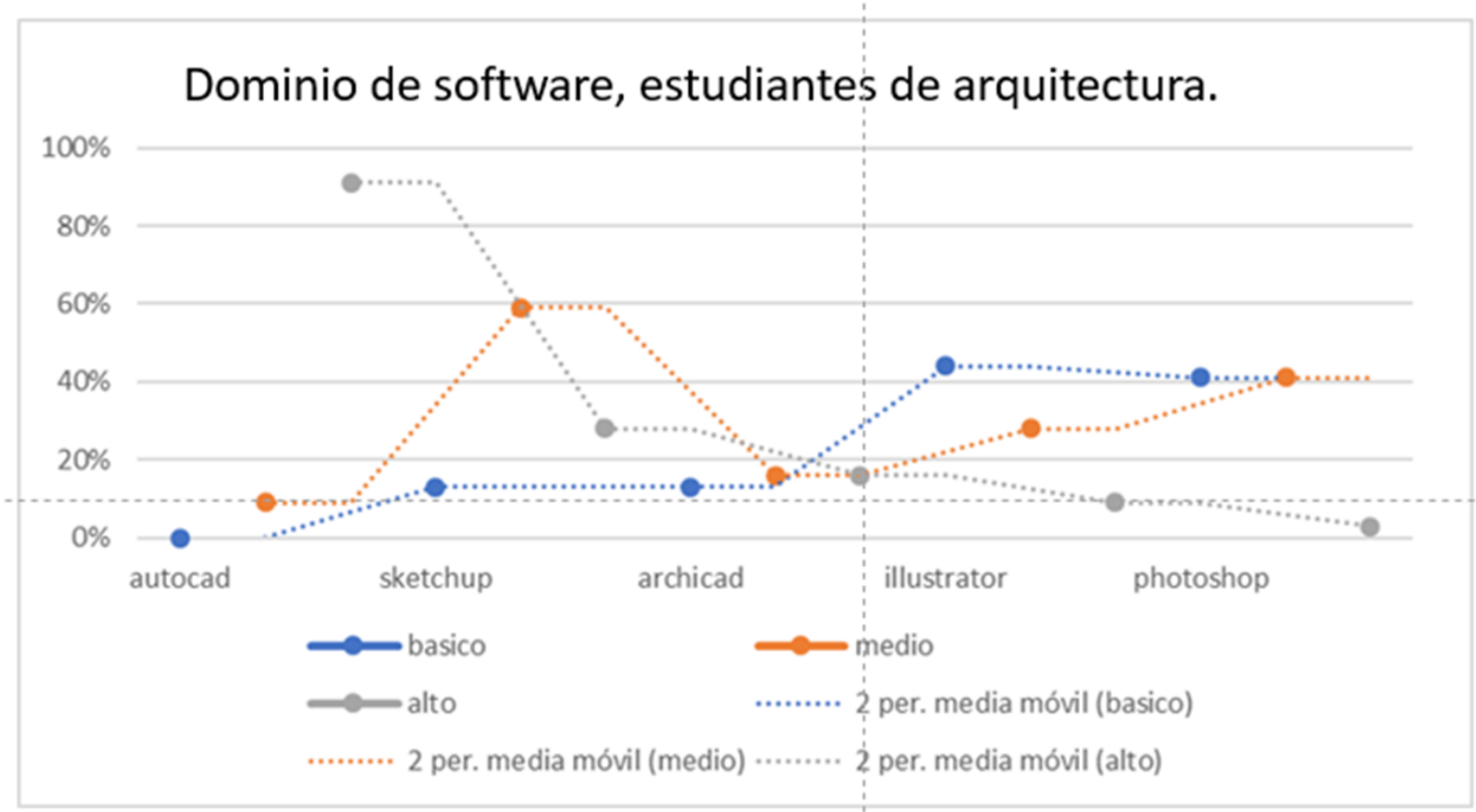

A través del dibujo hay una intención, se comunica, se observa. Este tema es crucial ya que su desarrollo y desempeño jugo un importante papel en el concurso del Banco Mundial. Según el plan de estudios vigente todo está centrado durante los primeros niveles, donde se utiliza y se fomenta la utilización de los medios de 
representación "analógicos" (maquetas y dibujos a mano) ya que la serie de recursos gráficos que tienen a su alcance los estudiantes para transmitir sus ideas arquitectónicas durante las etapas de formación iniciales, suelen ser escasos y muchas veces, interiorizarse tempranamente con programas de modelado y renderizado acorta los tiempos de desarrollo de ideas y podría representar una dificultad si no se tiene una buena formación en geometría. En dicha etapa se vuelve más relevante "aprender a pensar en el espacio arquitectónico" que "aprender a representarlo". Ello implicaría un inmenso trabajo para elegir, diseñar y utilizar las herramientas tecnológicas adecuadas para todo el proceso de formación del arquitecto desde los niveles básicos hasta los avanzados.

Una evidencia de esto la encontramos en la búsqueda de un prototipo que utilice el menor tipo de paneles para abaratar costos, con importantes restricciones a la hora de cerrar los perímetros geométricos, a tal punto que debió utilizarse la figura de un comodín para cerrar los perímetros, pero de una manera muy artesanal dibujando cada panel y sorteando los obstáculos, para ello se diseñaron básicamente dos tipos de paneles, estructurales y de pared, con dificultades se encontraron los cierres de los perímetros geométricos de la implantación del prototipo que fueron presentados en el concurso del Banco Mundial.

El experimento de Arquitectura, a pesar de las valoraciones de ciertos indicadores que representaron dificultades, alcanzó niveles de fortaleza en aspectos tales como: resolución de problemas, planificación, organización, focalización de atractores, velocidad de la investigación, que permitieron obtener el certificado de finalista en el concurso Resilient Homes, Desing Challenge, al proyecto LEGOS P otorgado por el Banco Mundial, Uno-Habitat, Build Academy, Airbnb, GFDRR, el 14 de Diciembre del 2018.

Finalmente, los resultados obtenidos en el proyecto de investigación apuntan a una revisión global del plan de estudios de la carrera de arquitectura, desde la perspectiva de la epistemología de la arquitectura, que permita articular los ejes de producción de conocimiento que se fundamentan en el "proceso proyectual de la experimentación del espacio"; ello nos conduce inexorablemente, a definir los diferentes pensamientos que debemos formular, pensamiento arquitectónico, pensamiento geómetra-digital, pensamiento artístico, pensamiento constructivo., que son los pensamientos que necesitamos construir para producir conocimientos que exige el proceso proyectual, provocando la reestructuración de las áreas y la formulación de nuevos pesos de las áreas en el conjunto del plan donde la geometría se constituya en unos de los elementos articuladores (ver Figura 6). 
Figura 6

Propuesta Curricular para la Carrera de arquitectura 2017

PROPUESTA CURRICULAR PARA LA CARRERA DE ARQUITECTURA - UG MARZO 2017

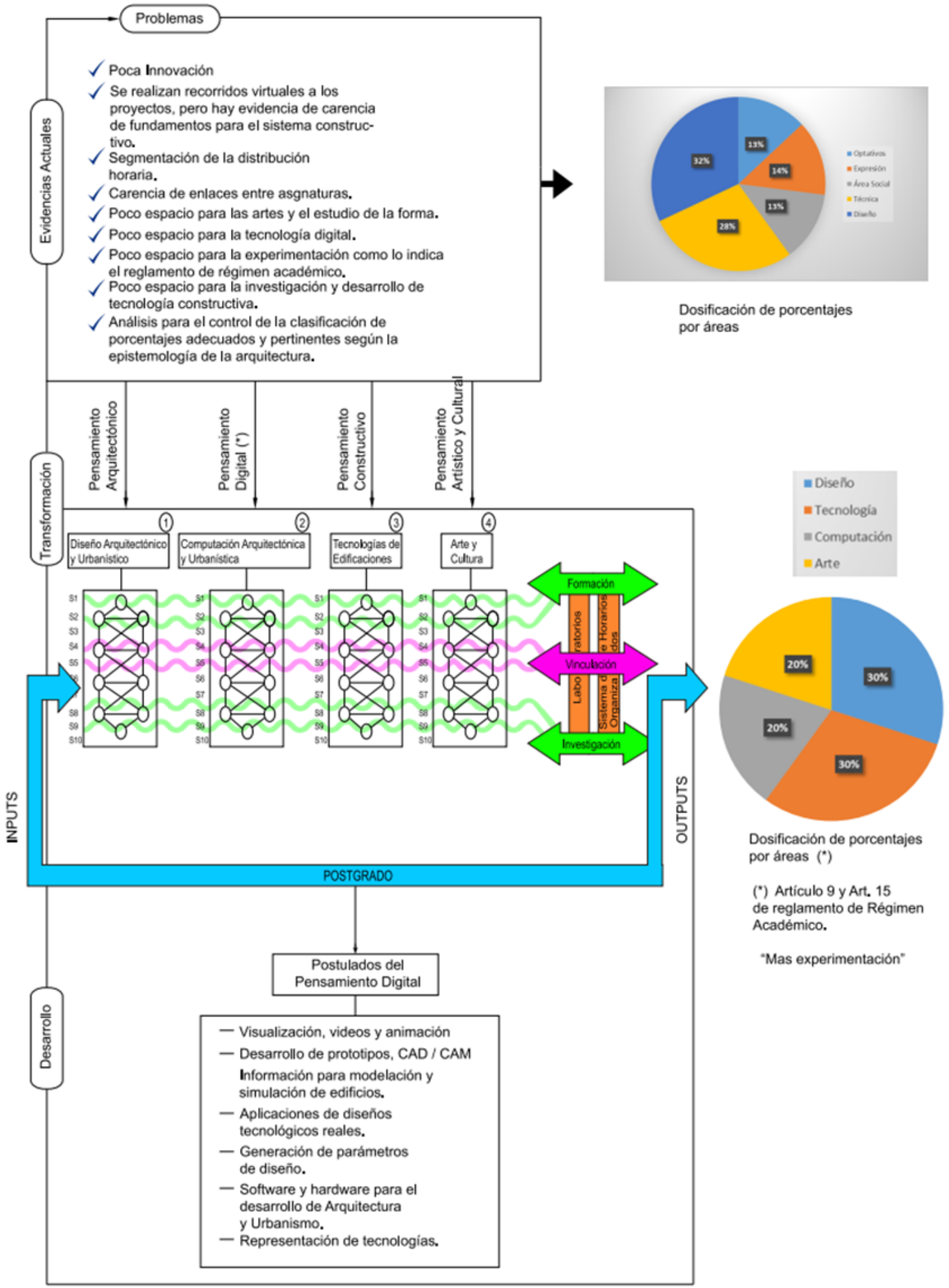


La propuesta curricular para la carrera de arquitectura y la propuesta de living lab para el taller de proyectos, se articularía plenamente en lo que dice el Art.8, literal b) y Art.15 del Reglamento de Régimen Académico (Consejo de Educación Superior del Ecuador, 2017) que hacen referencia a la fase de experimentación;

a) Ingenierías y Arquitectura. Forman profesionales capaces de aplicar las ciencias básicas y usar herramientas metodológicas para la solución de problemas concretos, mediante el diseño, perfeccionamiento, implementación y evaluación de modelos y estrategias de innovación tecnológica.

b) Actividades de aprendizaje. Están orientadas al desarrollo de experiencias de aplicación de los aprendizajes. Estas prácticas pueden ser, entre otras: actividades académicas desarrolladas en escenarios experimentales o en laboratorios, las prácticas de campo, trabajos de observación dirigida, resolución de problemas, talleres, manejo de base de datos y acervos bibliográficos. La planificación de estas actividades deberá garantizar el uso de conocimientos teóricos, metodológicos, y técnicos instrumentales y podrá ejecutarse en diversos entornos de aprendizaje. Las actividades prácticas deben ser supervisadas y evaluadas por el profesor, el personal técnico docente y los ayudantes de cátedra y de investigación.

Como se podrá observar, según este reglamento, es imperativo desarrollar actividades experimentales espaciales, en los foros, en la malla, en el grado y en las áreas que garanticen y generen mayor impacto en la formación del arquitecto, sin embargo, el núcleo de la teoría y práctica de la arquitectura se fundamenta en el proceso proyectual de la experimentación del espacio, esto es asignarle el valor clave que le corresponde a la geometría, por lo que el reglamento debe apelar de forma más rigurosa a la naturaleza de esa práctica como fundamento científico para poder alimentar el proceso proyectual de la experimentación del espacio. De lo contrario, será solo una declaración reglamentaria inoficiosa porque no condiciona el valor científico de la práctica. Recordemos que la observación y los instrumentos son muy importantes a la hora de describir un evento para validar una teoría científica.

\section{Conclusiones}

La enseñanza de la arquitectura se fundamenta en una serie de relaciones de procesos para facilitar la comprensión del espacio arquitectónico, desde el pénsum de la carrera, pasando por los sylabos y sus contenidos, pero sobre todo, en el enfoque conceptual de la carrera. Por ello este trabajo trata sobre la reinvención de esas relaciones. La reinvención requiere que mejoremos nuestra comprensión del espacio arquitectónico como resultado del manejo de la geometría una vez que han evolucionado las tecnologías de representación digital del espacio 3D. Es indispensable reformular la formación del arquitecto fundamentalmente en el campo del dominio de la geometría frente a la tecnología.

Se pasó rápidamente del rapidógrafo a la computadora sin realizar las adaptaciones sistémicas que requiere utilizar nuevas herramientas para el diseño que exigen un proceso de experimentación de métodos y técnicas de lectura y elaboración gráfica para la generación de la forma. Una de las principales adaptaciones que deben realizarse tiene que ver con el papel de la geometría en los softwares. Y justamente la actual malla de arquitectura carece de esa armonización con las actuales maneras de comprender el espacio en el marco de los nuevos conocimientos de la geometría, los ecosistemas, nuevas modalidades de la enseñanza como los living lab, y otros dominios de la ciencia como la economía, la sostenibilidad y la organización humana.

El prototipo de vivienda de tecnología de paneles es un proyecto que se desarrolló en la modalidad living lab, para la convocatoria del concurso del Banco Mundial, que dependió de varios factores claves : el componente académico que la Facultad de Arquitectura suministra conocimientos a través de su malla curricular, el componente profesional que permitió afinar los resultados de los bloques de conocimientos. 
La propuesta consistió en proponer un prototipo de vivienda económica con paneles, para poblaciones asentadas en sitios vulnerables, siendo relevante el papel que desempeña la geometría a la hora de buscar la forma espacial más económica, para construirla en 72 horas, recurriendo a manuales y estudios de casos, encontrandose 5 tipos de paneles para el armado del prototipo, teniendo como puntos de partida los fundamentos de los juegos LEGO Papert de Investigación de Aprendizaje en el MIT Media Lab. El Grupo de Fabricación de Diseño que explora la aplicación de la fabricación digital de un edificio. Nuestro objetivo es descubrir métodos efectivos para aplicar el cálculo al diseño y producción de edificios directamente desde modelos 3D. Pero el proceso de fabricación del prototipo siguió la línea de producción Ford. La línea de fabricación debió dividirse en 16 bloques de tareas, siendo imprescindible la participación de profesores y estudiantes. Las prácticas profesionales, jugaron un papel importante, es un indicador binario porque permite medir el grado de eficacia y participación de los alumnos en el proyecto, además mide la participación colectiva en la resolución de los problemas. Para ello se utilizaron los informes o actas de cumplimento de metas y tareas grupales e individuales, concluyéndose que las tareas fueron cumplidas $80 \%$.

Los más importante que se logró con esta modalidad fue la construcción colectiva del conocimiento, a pesar que cada grupo de alumnos tenía una tarea, era imprescindible la conexión de tareas, y esto enriqueció los conocimientos de los alumnos en tiempo real, ya que no era necesario recibir clases en otras aulas, todo el proceso de fabricación se dio en el laboratorio living lab LEGOS P.

En el componente de profesionalización, los alumnos recibieron capacitaciones en las áreas de tecnología de la representación del espacio, sostenibilidad y optimización de recursos, materiales, y economía y producción en masas basándose los principios de Tylor y Ford. Para los ensambles se recurrió a los estudios de ensambles de la arquitectura de MAKOTO MASUZAWA, también era muy importante entender el concepto MIUS, technology assessment of Modular Integrated Utility Systems. Del concepto MIUS, proporcionar los servicios de utilidad deseados de acuerdo con el uso reducido de los recursos naturales críticos, la protección del medio ambiente y el costo mínimo. Del mismo modo las capacitaciones abordaron el concepto In-Situ Resource Utilization, Utilizacion Optimización de recursos in situ. Y desde luego la capacitación sobre los MATERIALES, investigación de materiales, fenoles, poliuretano expandido, pulioretano entre otros.

El trabajo interactivo que se logró con los estudiantes y los profesores tutores profundizó la relación necesaria para desarrollar los puntos fuertes y débiles del proceso en la fabricación del prototipo, y plantear las medidas necesarias para resolver los problemas.. Para ello se realizó una evaluación de 10 indicadores.

Las limitaciones que se encontraron en la producción del prototipo tienen que ver con la poca fortaleza que aporta el plan de estudios en la formación del arquitecto en la formación geómetra, y en la poca actualización del plan de estudios con la nueva generación de tecnologías para la representación del espacio. En pocos casos se encontró inasistencia de los estudiantes al laboratorio, y pocas señales de inasistencia del profesorado al proceso de monitoreo de las tareas estudiantiles. Otras de las dificultades que se encontraron fue la suspensión de la implementación de las 240 horas de práctica preprofesional que permitía a los estudiantes tributar sus prácticas al área de investigación para ser aprobadas para obtener el título de "profesional", debido a un reglamento que exige un condicionamiento de notas a los participantes.

El proyecto fue muy difundido dentro y fuera de la universidad, no obstante, se presentó una variable política importante, las nuevas autoridades de la Facultad le restaron apoyo al proyecto para su continuación. Sin embargo, la autoridad principal de la Universidad de Guayaquil dió el respaldo al proyecto de investigación, incluso confirió certificados a los participantes del proyecto por el logro alcanzado en el concurso del Banco Mundial. 
Los resultados obtenidos en el proyecto de investigación, apuntan a una revisión global del plan de estudios donde la formación geómetra-digital, sea un punto central de trabajo y discusión.

Tal enfoque permitirá incorporar el área de pensamiento digital, actualizando la formación de geometría en el marco de las nuevas tecnologías de representación del espacio digital. Además de incluir en la enseñanza del taller de proyectos la modalidad de living lab, para que el enfrentamiento de un proyecto arquitectónico este integrado por un grupo de asignaturas y no de forma aislada, como se evidencia en las mallas curriculares actuales; del mismo modo se recomienda que los proyectos de investigación deberían incluir esta modalidad, que podría recomendarse realizar reformas del Reglamento de Régimen Académico (RRA) propuesta por el Consejo de Educación Superior del Ecuador. Esto implicaría que para el taller de proyectos deberían concurrir varias asignaturas entre ellas las que utilicen software básico para dibujar y software especializado para el tratamiento de casos, simuladores, etc, estudio de materiales, construcciones y estructuras, entre otras. Los talleres de proyectos se convertirían en cluster de asignaturas.

\section{Referencias bibliográficas}

Agudo, J. (2017). Sostenibilidad y fabrication laboratory aplicado a la arquitectura: Neil A. Gershenfeld y el how to make (almost) anything:

https://www.researchgate.net/publication/331233695_Sostenibilidad_y_Fabrication_Laboratory_aplicado_a_I a_Arquitectura_Neil_A_Gershenfeld_y_el_How_to_Make_Almost_Anything

Banco Mundial. (2019). Winners of «resilient homes design challenge» announced: https://www.worldbank.org/en/topic/disasterriskmanagement/brief/resilient-homes-challenge. World Bank, (2019). RESILIENT HOMES DESIGN CHALLENGE. Washington, DC: World Bank.: http://viewer.zmags.com/publication/858e96ae\#/858e96ae/143

Bertalanffy, L. (1999). Teoría general de sistemas de Ludwig Von BErtalanffy[. UNAM.: http://computo.ceiich.unam.mx/webceiich/docs/libro/Teoria_General_de_Sistemas_de_Ludwig_Von_Ber talanffy.pdf.

Biagioli, F. (2016). Space as a source of knowledge and as an object of research: The Transformation of the Concept of Space in the Post-Kantian Philosophy of Geometry.

https://www.academia.edu/30578143/Space_as_a_Source_of_Knowledge_and_as_an_Object_of_Resear ch_The_Transformation_of_the_Concept_of_Space_in_the_Post_Kantian_Philosophy_of_Geometry

Candela, F. (1975). Influencia de la tecnología en la creatividad arquitectónica: https://www.coam.org/media/Default\%20Files/fundacion/biblioteca/revista-arquitectura-100/19932000/docs/revista-articulos/revista-arquitectura-1995-n303-pag60-66.pdf

Castañeda, C. A. T. (2017). Construcciones sostenibles y certificaciones leed enColombia. 110: https://repository.uamerica.edu.co/bitstream/20.500.11839/7034/1/465230-2017-I-GA.pdf

Consejo de Educación Superior del Ecuador.(2017).reglamento de régimen a cadémico delconsejo de educación superior. Registro Oficial Edición Especial 854 de 25-ene.-2017: https://www.ces.gob.ec/lotaip/2018/Enero/Anexos\%20Procu/An-lit-a2Reglamento\%20de\%20R\%c3\%a9gimen\%20Acad\%c3\%a9mico.pdf

Correal, G. (2007). El proyecto de arquitectura como forma de producción de conocimiento. Revista de Arquitectura: .https://editorial.ucatolica.edu.co/index.php/RevArq/article/view/796

Darwin, C. (2009). El origen de las especies por medio de la selección natural. Universidad Nacional Autónoma de México; Consejo Superior de Investigaciones Científicas;Academia Mexicana de la Ciencias; Los libros 
de la Catarata.https://editorial.csic.es/publicaciones/libros/11715/978-84-00-08828-6/el-origen-de- lasespecies-por-medio-de-la-seleccio.html

Ehrlich, P. R., \& Holdren, J. P. (1971). Impact of Population Growth. Science, 171(3977), 1212-1217: https://science.sciencemag.org/content/171/3977/1212

Einstein, A., Lorentz, H. A., Minkowski, H., \& Weyl, H. (1952). THE PRINCIPLE OF RELATIVITY: Acollection of original memoirs on the special and general theory of relativity. DoverPublications: https://www.scirp.org/\%28S\%28vtj3fa45qm1ean45vvffcz55\%29\%29/reference/referencespapers.aspx?ref erenceid $=1645341$

Eriksson, M., Niitamo, V.-P., Kulkki, S., \& Hribernik, K. A. (2006). Living labs as a multi-contextualr\&d methodology. 2006 IEEE International Technology Management Conference (ICE), 1-8. https://doi.org/10.1109/ICE.2006.7477082

Espinoza, F., Reyes, L., Carbajo, A., Torres, R., \& Rendón, I. (2018). The fundamental algorithms that support the spatial geometry for the development of the technology of "P-LEGOS"'"panels applicable to affordable housing». /issues/fundamental-algorithms-support-spatial-geometry-development-technology-p-legospanels: http://webcache.googleusercontent.com/search?q=cache:LCaphTKGJIYJ:journalijcir.com/archive/201809 \&hl=es\&gl=ec\&strip $=1 \& v w s r c=0$ : file:///D:/MACRO\%20ARCHIVO/RESPALDO\%20ARCHIVO\%20MACRO\%20PARCIAL\%20HASTA\%2019\%20DE \%20FEBRERO/arcivos\%20pdf\%206\%20de\%20oct/articulo\%20cientifico\%20the\%20fundamental\%20algorit hms\%20that\%20support\%20the\%20spatial\%20geometry.pdf

Facultad de Arquitectura (2013-2014). Malla curricular. https://ugyemy.sharepoint.com/:b:/g/personal/felipe_espinozao_ug_edu_ec/EaiFZyFQCPxNIOwrsxreihkBaKGQraS6Pch gE1rhc3b3uA?e=dEWJLn https://ugyemy.sharepoint.com/:b:/g/personal/felipe_espinozao_ug_edu_ec/EVaLup_r4ztPsEUJAq5sZnkB1ZCq2EVz4J MYOX9abVN7sA?e=x08HGt

Fernández, A. (2017). La experiencia matemática de einstein: https://www.um.es/acc/la-experienciamatematica-de-einstein/

Ferran, S. (2016, mayo 3). Tecnología y desigualdad. La concentración de riqueza en la economía digital. CCCB LAB: https://lab.cccb.org/es/tecnologia-y-desigualdad-la-concentracion-de-riqueza-en-la-economiadigital/

Freitas-Lemes, P., Vilela, D., Germano, J. S., Santos, R., \& Sakuragi, J. (2017). Uso do software wingeom no processo de ensino aprendizagem de geometria. Revista ESPACIOS, 38(45): https://www.revistaespacios.com/a17v38n45/17384501.html

Galvão, E. T., Bonete, I. P., \& Caetano, J. J. (2016). Geometria no ensino fundamental: Relato de uma experência sobre a caracterização de figuras geométricas. Revista ESPACIOS / Vol. 37 ( $\left.N^{\circ} 29\right)$ : https://www.revistaespacios.com/a16v37n29/16372914.html

Gaumé, L. Á., \& Vázquez-Mozo, M. A. (2011). EINSTEIN Y LA GEOMETRÍA. REVISTA ESPAÑOLA DE FíSICA, 19(1): http://revistadefisica.es/index.php/ref/article/view/729/497 : https://arxiv.org/abs/physics/0503150

Guatura, D. S. da S., \& Romão, E. C. (2015). A importância do uso da tecnologia no ensino de geometria: planejamento e plano de aula. Revista ESPACIOS / Vol. 36 (No 13) Año 2015:

https://www.revistaespacios.com/a15v36n13/153613E1.html 
Hugo, H., Espinoza, F., Morales, I., Ortiz, E., Pérez, S., \& Salcedo, G. (2018). DELTA PROJECT:TOWARDSA SUSTAINABLE CAMPUS. SUSTAINABILITY, 10(10), 3695. https://doi.org/10.3390/su10103695

Kauffman, S. A. (2015). Antichaos and adaptation biological evolution may have been shapedby more thanjust natural selection. Computer models suggest that certain complex systems tend toward selforganization: https://www.semanticscholar.org/paper/Antichaos-and-Adaptation-Biological-evolutionmay-.-Kauffman/4f9e2eb52d464cdc44a6b69eca5142ab2280baa4

Maddison, A. (2007). Contours of the World Economy 1-2030 AD: Essays in Macro-Economic History. OUP Oxford: https://recyt.fecyt.es/index.php/IHE/article/view/70219/42452

Mandelbrot, B. B. (1997). La geometría fractal de la naturaleza. Tusquets Editores: https://ia802704.us.archive.org/4/items/pdfy-IAHD8SOkMGrTE8g6/138416567-Mandelbrot-Benoit-LaGeometria-Fractal-de-La-Naturaleza.pdf

Marshall, W. C., Girón, A., Correa, E., Rodríguez, P., Cabrera Morales, S., Vidal, G., \& Martínez, I.(2009). JOHN KENNETH GALBRAITH: homenaje en el centenario de su natalicio. Ola Financiera, 2(2): http://www.olafinanciera.unam.mx/new_web/02/pdfs/JohnKGalbraith-ReporteEspecial-OlaFin-2.pdf

Martín, J. (2011). Sysware. Dinámica de sistemas. Conceptos:https://libreria.tirant.com/es/libro/syswaredinamica-de-sistemas-conceptos-juan- martin-garcia-9788460924623

Muntañola, J. (1974). Presente y futuro de la epistemología de la arquitectura: file://C:/Users/feoco/Downloads/111835-Text\%20de\%20l'article-161131-1-10-20081029.pdf

Muñoz, V. (2019). El espacio arquitectónico: http://152.74.17.92/bitstream/11594/1769/1/el_espacio_arquitectonico.Image.Marked.pdf

Odum, H. T., \& Pinkerton, R. C. (1955). Time's speed regulator: The optimum efficiency for maximum power output in physical and biological systems. American Scientist, 43(2), 331-343: https://orrerysoftware.webs.com/02-Interests/02-C-Biophysical-Economics/02-C-01MPP\&MEPP/1955\%200dum,\%20Pinkerton\%20-\%20MPP.pdf

Pagel, M. (2008). Rise of the digital machine. Nature, 452(7188), 699: https://www.nature.com/articles/452699a

Pribram, K. H. (1981). El funcionamiento holonomico del cerebro: https://www.redalyc.org/pdf/805/80513201.pdf

Sanders, G. B. (2005). NASA, In-Situ Resource Utilization (ISRU) Capability Roadmap Executive Summary. May 13: http://www.marsjournal.org/contents/2006/0005/files/SandersDuke2005.pdf

Silva, J. M. da, Gonçalves, D. de C., Neves, I. C., Deus, J. V. de, Cota, K. G., \& Ferreira, W. L. (2018). Um estudo de áreas de telhados usando conceitos geométricos e recursos computacionais.Revista ESPACIOS, 39(14) https://www.revistaespacios.com/a18v39n14/18391411.html

Souza, M. L. de, \& Luccas, S. (2015). Geometria hiperbólica: Possibilidades para o ensino do conhecimento geométrico não euclidiano. Revista ESPACIOS / Vol. 36 ( $\left.\mathrm{N}^{\circ} 12\right)$ : https://www.revistaespacios.com/a15v36n12/153612E1.html

Tapia, V. (2013). Geometría de las superficies. Aplicaciones en física. Universidad Nacional de Colombia: https://sites.google.com/a/clusik.space/madisonhughi/geometria-de-las-superficies-aplicaciones-en-fisica

Torres, J. L. C. (2019). El club de Roma, aportes para el desarrollo. una historiografía de su obra publicada: https://trienal.fau.ucv.ve/2017/publicacion/articulos/AS/extenso/TIFAU2017_Extenso_AS02_JCasique.pdf

Esta obra está bajo una Licencia Creative Commons Attribución-NoCommercial 4.0 International 